\title{
Studies on the In Vivo and In Vitro Distribution of Apolipoprotein A-IV in Human Plasma and Lymph
}

\author{
Takao Ohta, Noel H. Fidge, and Paul J. Nestel \\ Cardiovascular Metabolism and Nutrition Research Unit, Baker Medical Research Institute, Prahran, Victoria 3181, Australia
}

\section{Abstract}

To investigate the unique distribution in plasma of apolipoprotein A-IV (apo A-IV) we have determined, in a series of in vitro and in vivo studies, the redistribution among lipoproteins of ${ }^{125}$ I-apo A-IV.

Free ${ }^{125}$ I-apo A-IV associated predominantly with high density lipoprotein (HDL) $(72 \pm 3.5 \%)$ in incubations with plasma, and with triglyceride-rich lipoproteins (TRL) $(65 \pm 3.0 \%)$ in incubations with lymph, rather than with the lipoprotein-deficient fraction (LDF) where $>\mathbf{9 0} \%$ of apo A-IV resides. Incubations with ${ }^{125}$ I-apo A-IV (incorporated within HDL or TRL) also resulted in similar redistributions of label. Specific radioactivities of apo A-IV in HDL and in TRL were of a similar order and 15-fold higher than those in LDF. However, when ${ }^{125}$ I-apo AIV in LDF was incubated with plasma, $57 \pm 2.6 \%$ of label remained in the LDF, though the specific radioactivity of apo AIV in HDL was 15-fold higher than in LDF. Thus, apo A-IV apparently exchanges freely between TRL, HDL, and a part of apo A-IV in LDF, but most of apo A-IV in LDF is refractive to free exchange or transfer.

In vivo experiments carried out in five subjects, in which ${ }^{125} \mathrm{I}$ apo A-IV was injected within TRL, HDL, or LDF, were consistent with the in vitro data in showing rapid exchange of label among plasma apo A-IV containing fractions with much higher specific radioactivities in HDL than in LDF (10-30-fold). However, the small fraction of apo A-IV in LDF that did become labeled was removed from plasma in a biexponential fashion and at the same rate as from HDL.

Thus, only a small fraction of the bulk of apo A-IV in plasma LDF exchanges freely with apo A-IV in TRL and HDL, suggesting that apo A-IV in LDF exists in at least two pools. This is consistent with our previous findings that apo A-IV in plasma is present in two distinct complexes with lipids and other peptides.

\section{Introduction}

The plasma protein apolipoprotein A-IV (apo A-IV) ${ }^{1}$ of molecular weight 46,000 has achieved the status of an apolipoprotein

Dr. Ohta is a visiting scientist from the Department of Pediatrics, Kumamoto University Medical School, Kumamoto 860, Japan.

Address correspondence to Dr. Fidge.

Received for publication 30 April 1984 and in revised form 10 April 1985

1. Abbreviations used in this paper: apo A-IV, apolipoprotein A-IV; HDL, high density lipoprotein; LDF, lipoprotein-deficient fraction; TRL, triglyceride-rich lipoproteins; VLDL, very low density lipoprotein.

J. Clin. Invest.

(C) The American Society for Clinical Investigation, Inc.

$0021-9738 / 85 / 09 / 1252 / 09 \$ 1.00$

Volume 76, September 1985, 1252-1260 on the basis that it is a major component of lymph chylomicrons in humans and in other species (1-5), and that in the rat it present in moderate amounts in high density lipoprotein (HDL) (2, 3, 6-9). Unlike most other apolipoproteins, however, apo AIV is not a major component of human plasma lipoproteins, and although present in moderate concentrations of between 14 and $30 \mathrm{mg} / \mathrm{dl}(5,10)$, most of the apo A-IV exists in the lipoprotein-deficient fraction (LDF) of plasma $(d>1.21 \mathrm{~g} / \mathrm{ml})$. Recent studies in this laboratory have shown, however, that even in the fraction with $d>1.21 \mathrm{~g} / \mathrm{ml}$ apo A-IV exists in association with lipids and other apolipoproteins in lipoproteinlike species comprising apo A-IV, phospholipid, cholesteryl ester, and triglyceride as well as apo A-I and a third peptide of $M_{\mathrm{r}} 59,000$ (11). This has raised the possibility that apo A-IV in the LDF of plasma may have functional relationships that are distinct from those of apo A-IV in HDL and chylomicrons.

Apo A-IV concentrations rise in plasma after the ingestion of fat (12), and we have found elevated levels in patients with chronic renal failure in association with the accumulation of chylomicron remnants (13). Its presence in high concentration in lymph chylomicrons of both humans $(5,12)$ and experimental animals (14) suggests that it may play either a structural or metabolic role, or both, in triglyceride transport. Recent studies have suggested it may also be involved in cholesterol transport since, in rats, the distribution of apo A-IV between HDL and the LDF of plasma is influenced by feeding cholesterol (8).

The unusual distribution and metabolism of the A-IV apolipoprotein may provide a clue to its function. It is important therefore to determine the manner in which the peptide exchanges or transfers between different human plasma fractions, and in this paper we report studies on the redistribution of apo A-IV in human plasma, in two sets of experiments. Firstly, the distribution of radiolabeled apo A-IV was determined in vitro during incubations with plasma or lymph. Secondly, the distribution of radiolabeled apo A-IV was followed in vivo within plasma over $2 \mathrm{~d}$ after infusing labeled apo A-IV to determine the physiological compartmentalization of apo A-IV in plasma.

\section{Methods}

Isolation of apo A-IV. Apo A-IV was isolated from the LDF of plasma and human lymph triglyceride-rich lipoproteins (TRL) as described before (11). Briefly, lipoprotein-deficient plasma was prepared by removing lipoproteins of $d<1.25 \mathrm{~g} / \mathrm{ml}$ by ultracentrifugation and dialyzed against $0.15 \mathrm{M} \mathrm{NaCl}$ containing $1 \mathrm{mM}$ EDTA buffered to $\mathrm{pH} 7.4$ with Trizma base (Sigma Chemical Co., St. Louis, MO) (buffer A).

The dialyzed LDF was mixed with 0.3 vol artificial fat emulsion (Neutralipid; Pharmacia Fine Chemicals, Piscataway, NJ) and incubated at $37^{\circ} \mathrm{C}$ for $2 \mathrm{~h}$. After centrifugation in a rotor (model SW-41; Beckman Instruments, Inc., Fullerton, CA) for $30 \mathrm{~min}$ at $4^{\circ} \mathrm{C}$, the supernatant lipid layer was dispersed in buffer $\mathrm{A}$ to yield the original volume of Neutralipid and centrifuged twice further to remove unbound plasma protein. Finally, the lipid layer was resuspended in buffer A and delipidated with chloroform, methanol, and ether as described previously (15). 
The delipidated protein was immediately dissolved in $0.1 \mathrm{M}$ Tris $\mathrm{HCl}$, pH 7.5, containing $6 \mathrm{M}$ urea and subjected to chromatography on a column of QAE-Sephadex A-50 (Pharmacia Fine Chemicals). A linear gradient of $\mathrm{NaCl}$ was established between 0 and $0.3 \mathrm{M} \mathrm{NaCl}$ in $6 \mathrm{M}$ urea and $0.1 \mathrm{M}$ Tris $\mathrm{HCl}, \mathrm{pH}$ 7.5. The fraction richest in apo A-IV was pooled, dialyzed, and lyophilized. Final purification of apo A-IV was carried out using preparative gel electrophoresis on $10 \%$ polyacrylamide gels (in $0.1 \%$ sodium dodecyl sulfate [SDS]) in the presence of $\beta$-mercaptoethanol (3).

Preparation of antiserum. New Zealand White rabbits $(2.5-3 \mathrm{~kg})$ were immunized with $100 \mu \mathrm{g}$ of purified LDF apo A-IV. The protein solution was emulsified with an equal volume of Freund's complete adjuvant and injected intradermally into multiple sites on the backs of the rabbits. The injection was repeated intramuscularly three times at intervals of 2-3 wk, and animals were bled $8 \mathrm{~d}$ after the last injection. The antisera did not react with pure apo A-I, A-II, B, C-I, C-II, C-III, or E nor with albumin, but in immunoassay procedures appeared identical to antisera prepared against apo A-IV from lymph TRL. Apo A-IV isolated from LDF and lymph TRL reacted with lines of identity against apo A-IV antiserum in exactly the same manner as native apo A-IV within LDF, TRL, and HDL.

Apo A-IV Sepharose 4B affinity column. Purified apo A-IV from plasma LDF was dissolved and extensively dialyzed against $0.1 \mathrm{M}$ $\mathrm{NaHCO}_{3}$ containing $0.5 \mathrm{M} \mathrm{NaCl}$ (pH 8.0). Then apo A-IV was coupled to cyanogen bromide-activated Sepharose 4B (Pharmacia Fine Chemicals) at $\mathrm{pH} 8.0$, as described by Cuatrecasas (16), using a ratio of $1 \mathrm{mg}$ apo A-IV/ml of activated Sepharose 4B. Finally, apo A-IV Sepharose 4B was equilibrated with $0.1 \mathrm{M}$ borate containing $1.0 \mathrm{M} \mathrm{NaCl}$ and $0.1 \%$ Tween $20, \mathrm{pH} 8.0$, and transferred to chromatography columns.

Antiserum to apo A-IV was used for the isolation of apo A-IV-specific IgG. Antiserum, prepared as described above, was dialyzed against equilibrating buffer and applied to the apo A-IV Sepharose 4B affinity column. Material bound to the column was eluted with $0.2 \mathrm{M}$ glycine $/ \mathrm{HCl}, \mathrm{pH}$ 2.5 , and dialyzed against buffer $\mathrm{A}$. The eluate from the column showed a single band on SDS polyacrylamide gel electrophoresis and immunologically reacted only with apo A-IV.

Anti-apo A-IV Sepharose 4B column (A-IV immunoaffinity column). Apo A-IV-specific IgG was dialyzed against $0.1 \mathrm{M} \mathrm{K}_{2} \mathrm{HPO}_{4}$ containing $0.5 \mathrm{M} \mathrm{NaCl}(\mathrm{pH} 6.5$ ) and coupled to cyanogen bromide-activated Sepharose 4B as described by Cuatrecasas (16) using a ratio of $2 \mathrm{mg} \mathrm{IgG/}$ $\mathrm{ml}$ activated Sepharose 4B. Chromatography was performed on a column of $2.5 \times 30 \mathrm{~cm}$. Anti-apo A-IV Sepharose 4B was packed above twice the bed volume of Sephadex G-25 (Medium; Pharmacia Fine Chemicals) in $0.05 \mathrm{M}$ Tris containing $0.5 \mathrm{M} \mathrm{NaCl}, \mathrm{pH} 7.5$, to avoid prolonged interaction of the desorbing reagent with protein eluted from the column. Then the column was equilibrated with $0.05 \mathrm{M}$ Tris containing $0.5 \mathrm{M}$ $\mathrm{NaCl}$ and $0.1 \% \mathrm{NaN}_{3}$ at $\mathrm{pH} 7.5$.

Isolation of apo A-IV from human lymph TRL. TRL was isolated from the lymph obtained from the chylous pleural effusion of a patient with tuberous sclerosis with lymphangiomyomatosis of the thoracic duct. Centrifugation was carried out, without further adjustment of density in a 45- Ti rotor in the ultracentrifuge (model L 5-65B; Beckman Instruments, Inc.) for $5 \mathrm{~h}$ at $4^{\circ} \mathrm{C}$. After removal of the infranate by aspiration, the gel like creamy supernatant at the top was resuspended in $30 \mathrm{ml}$ of buffer $A$. The top fraction was recentrifuged for $6 \mathrm{~h}$ at $50,000 \mathrm{rpm}$ in a 50-Ti rotor (Beckman Instruments, Inc.). A portion of the lipoprotein was delipidated with chloroform, methanol, and ether as described above. The apolipoprotein moiety was dissolved in the equilibrating buffer (immunoaffinity column buffer), dialyzed against the same buffer for $16 \mathrm{~h}$, and applied to the anti-apo A-IV IgG immunoaffinity column. Application of the sample was followed by extensive washing with equilibrating buffer until the absorbance $(280 \mathrm{~nm})$ returned to the base line. Material bound to the column was eluted with $6 \mathrm{M}$ guanidine $\mathrm{HCl}, \mathrm{pH} 3.1$, and then dialyzed against buffer $\mathrm{A}$

Radioiodination of apo A-IV. Immunoaffinity-purified apo A-IV from lymph TRL $(0.1 \mathrm{mg}$ dissolved in $0.2 \mathrm{ml} 4 \mathrm{M}$ borate buffer, $\mathrm{pH} 8.0)$ was labeled with ${ }^{125} \mathrm{I}$ by the iodine monochloride technique (17). Efficiency of labeling was $\sim 25 \%$ and the iodine/protein ratio was $<1 \mathrm{~g} /$ atom iodine per mol protein. Removal of free ${ }^{125} \mathrm{I}$ was accomplished by gel filtration through Sephadex G-50 columns $(0.7 \times 20 \mathrm{~cm})$, equilibrated with buffer A followed by dialysis against the same buffer.

Analytical procedures. Disk gel electrophoresis in $15 \%$ polyacrylamide gels containing $0.1 \%$ SDS was performed according to the methods described by Weber and Osborn (18) using Tris $\mathrm{HCl}$ rather than phosphate buffer.

Isoelectric focusing was performed in polyacrylamide disk gels containing $8 \mathrm{M}$ urea and ampholytes (pH 4-6), essentially as described by Gidez et al. (19). Amino acid analysis was performed on an analyzer (model 6300; Beckman Instruments, Inc.). Samples were dialyzed against distilled water, lyophilyzed, and hydrolyzed in $6 \mathrm{~N} \mathrm{HCl}$ at $115^{\circ} \mathrm{C}$ for $24 \mathrm{~h}$ in evacuated sealed tubes.

To compare the amino acid composition of the major isoforms of apo A-IV, two-dimensional gel electrophoresis was performed as described previously (11). After staining and destaining the second dimension slab gels, the corresponding isoforms from four separations were pooled and the proteins electroeluted from the gel slices. The proteins were then precipitated with 9 vol of pure methanol, allowed to stand overnight at $-20^{\circ} \mathrm{C}$, and the apolipoproteins were then recovered by centrifugation at 2,000 rpm for $5 \mathrm{~min}$ at $4^{\circ} \mathrm{C}$, leaving Coomassie Blue and SDS in the methanol phase. After they were washed once with methanol, the peptides were dried under $\mathrm{N}_{2}$ and hydrolyzed as described above.

\section{In vitro studies}

Incubation of ${ }^{125} \mathrm{I}$-apo A-IV with plasma and lymph. The ${ }^{125} \mathrm{I}$-apo A-IV was mixed with either normal plasma $(7 \mathrm{ml})$, human thoracic lymph (7 $\mathrm{ml})$, or a mixture of lymph $(3.5 \mathrm{ml})$ and normal plasma $(3.5 \mathrm{ml})$ and incubated for $1 \mathrm{~h}$ at $37^{\circ} \mathrm{C}$ while being gently shaken. The samples were then applied to a column $(2.5 \times 90 \mathrm{~cm})$ of Sepharose CL-6B $(6 \%$ agarose gel; Pharmacia Fine Chemicals) equilibrated with buffer $A$. The flow rate was $21 \mathrm{ml} / \mathrm{h}$, and fractions of $4.2 \mathrm{ml}$ were collected. The column was operated at $6^{\circ} \mathrm{C}$. The tubes were monitored for absorbance at $280 \mathrm{~nm}$ and radioassayed to identify ${ }^{125} \mathrm{I}$-apo A-IV-containing fractions.

In further experiments labeled fractions obtained after gel filtration were reincubated with either plasma or lymph. Briefly, a portion $(2 \mathrm{ml})$ of ${ }^{125} \mathrm{I}$-apo A-IV lymph TRL and HDL from Sepharose CL-6B columns was mixed with plasma $(5 \mathrm{ml})$ or lymph $(5 \mathrm{ml})$. Similar reincubations were performed with the LDF (pooled from several columns and concentrated to obtain sufficient radioactivity). The mixtures were shaken gently at $37^{\circ} \mathrm{C}$ for $1 \mathrm{~h}$ and applied to a Sepharose CL-6B column in the same manner as described before. Altogether six sets of incubations were carried out as shown in Table II, each experiment having been performed three times.

Measurement of apo A-IV concentration. Apo A-IV concentration was determined by electroimmunoassay as described before (3). The antisera used in this study showed strong, clear rocket-type precipitation zones with pure apo A-IV (used as standards) or when apo A-IV was present in plasma or lipoprotein fractions. The immunoassay was sensitive at concentrations as low as $3 \mu \mathrm{g} / \mathrm{ml}$.

\section{In vivo studies}

Subjects. Five studies were carried out in which labeled apo A-IV in lymph TRL, plasma TRL, HDL, or LDF was injected intravenously. Each preparation was obtained from a separate subject and reinjected into the donor. The various fractions were separated by ultracentrifugation (20), and the apo A-IV within each fraction was radioiodinated by exchange with purified sterile and pyrogen-free ${ }^{125} \mathrm{I}$-apo A-IV. Each lipoprotein or LDF was incubated with 3-5 mg labeled apo A-IV (40-70 $\mu \mathrm{Ci})$ at $37^{\circ} \mathrm{C}$ for $1 \mathrm{~h}$ and then reisolated in the appropriate density range. Sterility and pyrogenicity were established before administration.

The details of the five subjects are given in Table IV. Briefly, lymph TRL was injected into a normolipidemic woman (no. 1) with a chylothorax, plasma TRL was injected into a man (no. 2) with hyperchylomicronemia (type 5 hyperlipoproteinemia), HDL was injected into a man (no. 3) with mild hypertriglyceridemia, and LDF was injected into a man (no. 4) with combined hyperlipoproteinemia and another (no. 5) with moderate hypertriglyceridemia. Written consent was obtained from each subject. 
In vivo distribution of apo A-IV. After the labeled preparation was injected, samples of blood were obtained frequently during the first $6 \mathrm{~h}$ and less often thereafter for 26-28 h as shown in Figs. 3-7. Normal food intake including fat was allowed. To process large numbers of plasma samples, lipoproteins were separated by ultracentrifugation rather than by gel filtration after preliminary experiments that demonstrated similar findings with the two methods. The ratios of the specific radioactivities of apo A-IV in HDL and in LDF were, respectively, 15 and 13 when the same sample was fractionated by gel filtration or by ultracentrifugation.

Plasma $(10 \mathrm{ml})$ was centrifuged at $4^{\circ} \mathrm{C}$ in the 50 -Ti rotor of an ultracentrifuge (Beckman Instruments, Inc.). Plasma TRL comprising both chylomicrons and very low density lipoprotein (VLDL) were removed, and HDL and LDF were sequentially isolated (21). The fractions were dialyzed and assayed for radioactivity and apo A-IV concentration. Recovery of apo A-IV radioactivity after ultracentrifugation was $83-91 \%$.

The apo A-IV concentration was determined by rocket electroimmunoassay, as described above. Adequate amounts were present in $\mathrm{HDL}$ and in LDF only. Specific radioactivity time curves were therefore possible only for those two fractions. Radioactivity associated with TRL apo AIV was present in sufficient concentration for accurate assay.

\section{Results}

Characterization of isolated apo A-IV. Fig. 1 demonstrates that the apo A-IV, which was subsequently used in the following in vitro incubations, was isolated in pure form from delipidated lymph TRL (Fig. $1 A$ ), though it comprised several major and minor isoproteins (Fig. $1 B$ ), which have been characterized in detail and reported elsewhere (11). In brief, a similar number of isoproteins were observed in lymph TRL, plasma HDL, or plasma LDF, and all were positively identified as apo A-IV isoproteins by immunoblotting techniques.

Additionally, the amino acid compositions were determined on five isoforms of apo A-IV after they were separated by twodimensional gel electrophoresis. Insufficient amounts of the other five minor isoforms were recovered for analysis. As demonstrated in Table I, each isoform analyzed showed a similar amino acid composition that was consistent with the proportions of amino acid residues present in native apo A-IV.

The preparations of apo A-IV that were isolated as described above and subsequently radioiodinated for use in the redistribution studies showed the physical and chemical characteristics

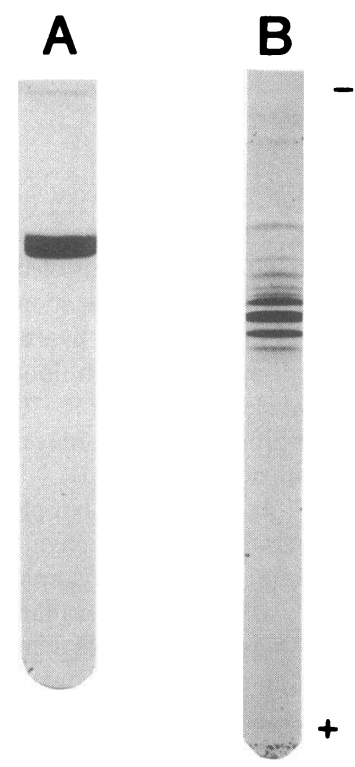

Figure 1. SDS polyacrylamide gel $(A)$ and isoelectric-focusing gel $(B)$ of apo A-IV isolated from human thoracic lymph TRL. $30 \mu \mathrm{g}$ of apo A-IV was applied to each gel. (-), Cathode site; $(+)$, anode site.
Table I. Amino Acid Composition of the Major Isoforms of Human Apo A-IV

\begin{tabular}{|c|c|c|c|c|c|}
\hline \multirow{2}{*}{$\begin{array}{l}\text { Amino } \\
\text { acid }\end{array}$} & \multicolumn{5}{|c|}{ Isoform* } \\
\hline & 1 & 7 & 8 & 9 & 10 \\
\hline & $\mathrm{mol} \%$ & $\mathrm{~mol} \%$ & $\mathrm{~mol} \mathrm{\%}$ & $\mathrm{mol} \%$ & $\mathrm{~mol} \%$ \\
\hline Asp & 10.7 & 10.6 & 10.1 & 10.2 & 12.4 \\
\hline Thr & 5.2 & 4.0 & 3.5 & 3.8 & 4.2 \\
\hline Ser & 5.5 & 8.7 & 7.7 & 7.1 & 9.9 \\
\hline Glu & 18.5 & 19.9 & 18.1 & 21.0 & 19.0 \\
\hline Pro & 4.4 & 3.5 & 3.5 & 2.9 & 3.7 \\
\hline Glył & 5.0 & 5.0 & 5.0 & 5.0 & 5.0 \\
\hline Ala & 9.3 & 7.2 & 7.8 & 7.5 & 7.1 \\
\hline Cys & ND & ND & ND & ND & ND \\
\hline Val & 6.9 & 5.8 & 6.3 & 5.3 & 5.6 \\
\hline Met & 0.9 & 1.1 & 1.2 & 0.8 & 0.5 \\
\hline Ile & 3.8 & 3.3 & 4.0 & 4.2 & 3.7 \\
\hline Leu & 10.8 & 11.2 & 14.3 & 13.2 & 10.7 \\
\hline Tyr & 1.1 & 1.0 & 0.9 & 0.4 & 1.0 \\
\hline Phe & 4.2 & 3.4 & 3.7 & 2.0 & 2.3 \\
\hline Lys & 1.6 & 2.3 & 1.8 & 1.6 & 2.3 \\
\hline His & 7.4 & 7.2 & 6.6 & 7.1 & 7.0 \\
\hline Arg & 4.9 & 5.8 & 5.7 & 5.8 & 5.6 \\
\hline Trp & ND & ND & ND & ND & ND \\
\hline
\end{tabular}

* Refers to isoform number (as identified in Fig. 7, reference 11). $¥$ Glycine values have been corrected for glycine present in the Tris glycine buffer by subtracting values obtained from control gels that had been exposed to buffer alone. The average corrected value using several controls was $5.0 \%$ for the five isoforms.

ND, Not detected.

of native apo A-IV according to several criteria, as follows. (a) Similar amino acid composition was shown for isolated labeled and unlabeled apo A-IV, values which agreed closely with those reported by other laboratories (11). (b) There was no evidence of aggregation or denaturation on the basis of similar electrophoretic mobilities on SDS polyacrylamide gels of isolated unlabeled or labeled apo A-IV and of native apo A-IV in lymph TRL. $(c)$ Full recovery of radioactivity of isolated, labeled apo A-IV was obtained within the apo A-IV band following polyacrylamide gel electrophoresis or with carrier apo A-IV following gel filtration. $(d){ }^{125}$ I-labeled apo A-IV, after incubation with the fraction of $d<1.21 \mathrm{~g} / \mathrm{ml}$ of plasma, associated completely with lipoproteins (TRL and HDL).

In vitro metabolism of apo A-IV. The major aim of the in vitro incubations was to determine the pattern of redistribution of radiolabeled apo A-IV among those plasma or lymph fractions that normally contain apo A-IV, TRL (VLDL and chylomicrons), HDL, and LDF. The series of incubations in which either plasma or lymph was incubated with either pure ${ }^{125} \mathrm{I}$-apo A-IV or ${ }^{125}$ I-apo A-IV previously incorporated into TRL, HDL, or LDF are shown in Table II and Figure $2 A-F$. The data shown was obtained using apo A-IV derived from lymph TRL, but similar results were obtained using apo A-IV derived from LDF. The Sepharose CL-6B columns were calibrated with lymph chylomicrons, plasma VLDL, LDL, HDL, and albumin to calibrate the volume of elution of each fraction as marked in Fig. 2. Recoveries of ${ }^{125} \mathrm{I}$-apo A-IV from the columns were $95.0 \pm 1.9 \%$.

The isolated ${ }^{125}$ I-apo A-IV associated predominantly with 
Table II. Redistribution of ${ }^{125}$ I-Apo A-IV in Fractions of Lymph and Plasma After Incubations of Whole Plasma or Whole Lymph with ${ }^{125}$ I-Apo A-IV Alone or ${ }^{125}$ I-Apo A-IV within a Lymph or Plasma Fraction

\begin{tabular}{|c|c|c|c|c|c|}
\hline \multirow[b]{2}{*}{ Experiment } & \multirow[b]{2}{*}{ Incubation mixture* } & \multicolumn{4}{|c|}{ Percent distribution } \\
\hline & & TRL & LDL & HDL & LDF \\
\hline 1 & ${ }^{125}$ I-Apo A-IV + plasma & $8 \pm 1.1 \ddagger$ & $5 \pm 1.0$ & $72 \pm 3.5$ & $14 \pm 2.0$ \\
\hline 2 & ${ }^{125} \mathrm{I}-\mathrm{Apo}$ A-IV + lymph & $65 \pm 3.0$ & $6 \pm 1.0$ & $16 \pm 2.0$ & $13 \pm 2.0$ \\
\hline 3 & ${ }^{125} \mathrm{I}$-Apo A-IV + plasma and lymph & $45 \pm 1.5$ & $14 \pm 1.5$ & $28 \pm 2.0$ & $13 \pm 0.5$ \\
\hline 4 & ${ }^{125} \mathrm{I}$-Apo A-IV in plasma HDL + lymph & $62 \pm 2.1$ & $5 \pm 0.7$ & $24 \pm 2.0$ & $9 \pm 0.5$ \\
\hline 5 & ${ }^{125}$ I-Apo A-IV in lymph TRL + plasma & $21 \pm 0.8$ & $8 \pm 2.1$ & $58 \pm 1.3$ & $13 \pm 2.0$ \\
\hline 6 & ${ }^{125} \mathrm{I}-\mathrm{Apo} \mathrm{A}-\mathrm{IV}$ in plasma LDF + plasma & $5 \pm 3.3$ & $8 \pm 1.7$ & $30 \pm 2.6$ & $57 \pm 2.6$ \\
\hline
\end{tabular}

* $n=3$ for each experiment. $\ddagger$ Mean \pm SD.

HDL in incubations with whole plasma, with TRL in incubations with whole lymph, or with both HDL and TRL in incubations with equal amounts of plasma and lymph (experiments 1-3).
In incubations with radioiodinated apo A-IV that had been initially incorporated into fractions of plasma or lymph (experiments 4-6), the redistribution of label after further incubations
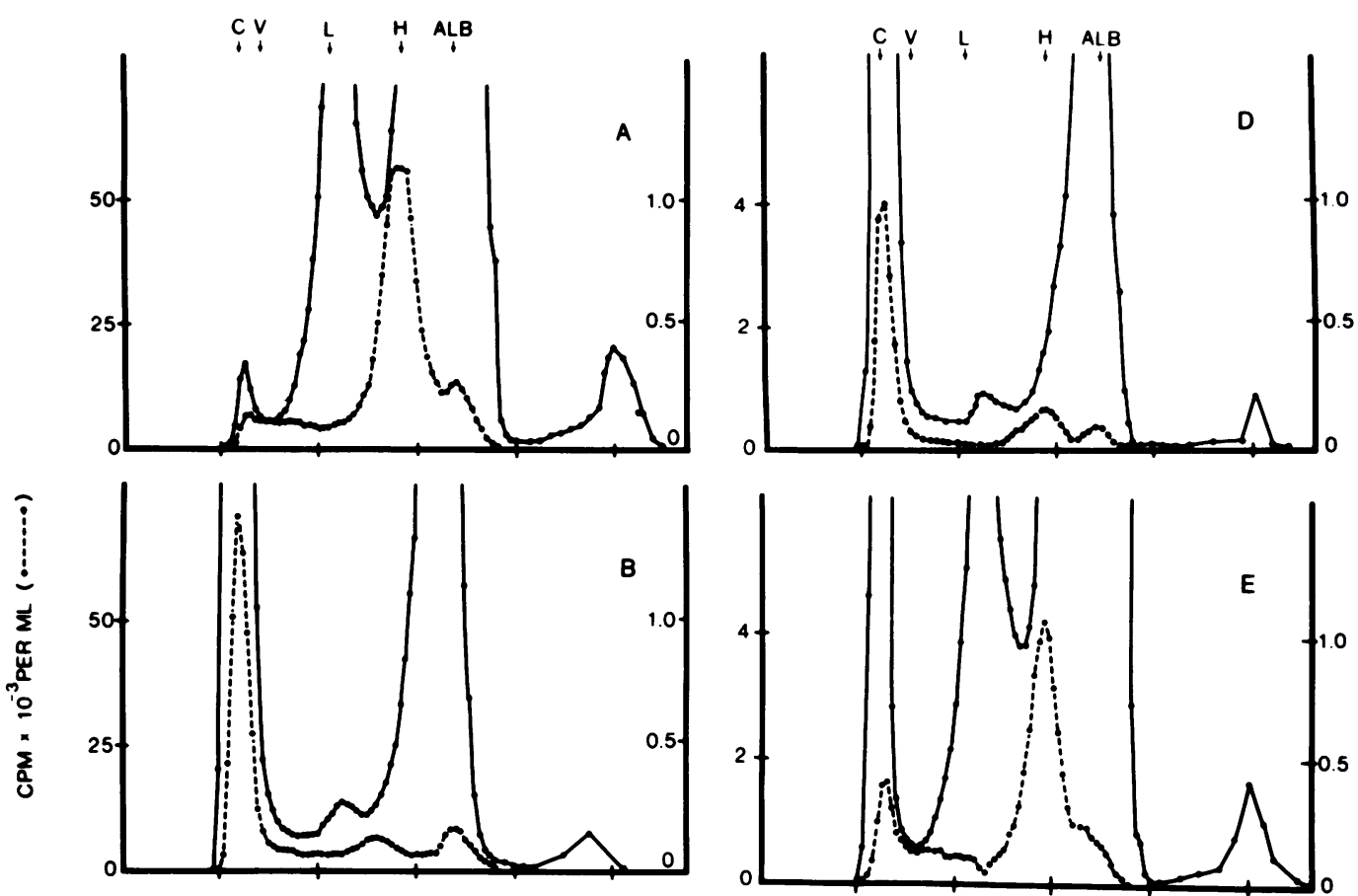

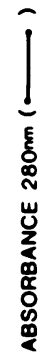
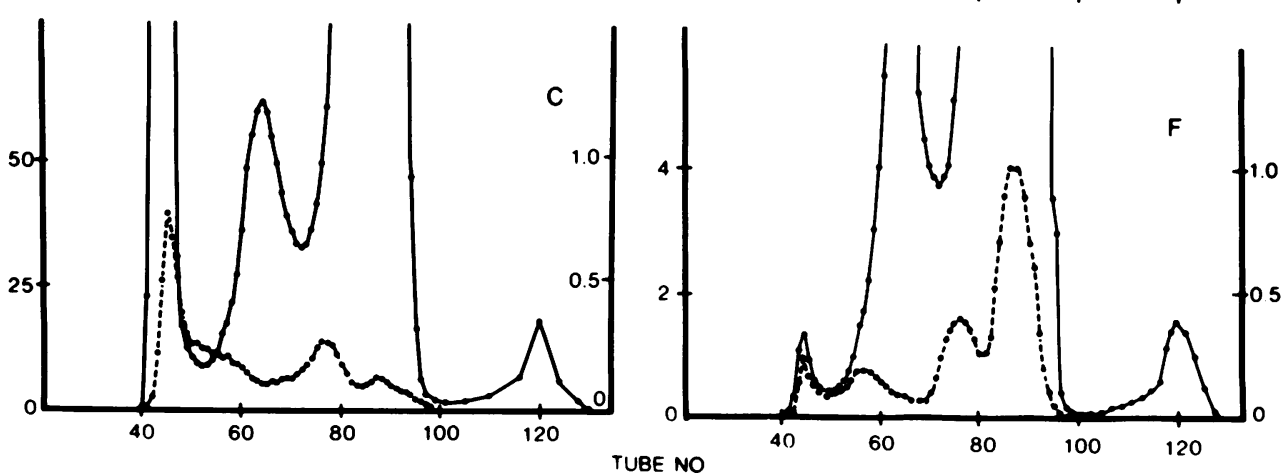

Figure 2. Distribution of ${ }^{125} \mathrm{I}$-apo A-IV after incubation with plasma, human lymph, and a mixture of lymph and plasma. Representative elution profiles from Sepharose CL-6B $(2.5 \times 90 \mathrm{~cm})$, operated as described in the text, are shown after incubation of ${ }^{125} \mathrm{I}$-apo A-IV with $A$, $7 \mathrm{ml}$ of plasma, $B, 7 \mathrm{ml}$ of lymph, and $C, 7 \mathrm{ml}$ of a mixture of lymph $(3.5 \mathrm{ml})$ and plasma $(3.5 \mathrm{ml})$. In other experiments, ${ }^{125}$ I-labeled (apo A-IV) lymph TRL, HDL, or LDF obtained from columns were rein-

cubated with lymph or plasma and elution profiles are shown in $D-F$. $D, 5 \mathrm{ml}$ of lymph incubated with $2.0 \mathrm{ml}{ }^{125} \mathrm{I}$-apo A-IV HDL; $E, 5 \mathrm{ml}$ of plasma incubated with $2.0 \mathrm{ml}$ of ${ }^{125} \mathrm{I}$-apo A-IV lymph TRL; and $F$, $5 \mathrm{ml}$ of plasma reincubated with $2.0 \mathrm{ml}$ of ${ }^{125} \mathrm{I}$-apo A-IV LDF (concentrated fourfold, as described in Methods). Elution volume of human chylomicrons (C), VLDL (V), LDL (L), HDL (H), and bovine serume albumin (ALB) are shown for references. 
mostly resembled that obtained with labeled apo A-IV alone. However, under these circumstances more label remained with the fraction with which it had become initially associated. Thus, for example, more ${ }^{125} \mathrm{I}$-apo A-IV was recovered in HDL when lymph was incubated with HDL-associated ${ }^{125} \mathrm{I}$-apo A-IV than with ${ }^{125} \mathrm{I}$-apo A-IV alone; however, with both incubations most of the label redistributed to the TRL fraction. The important exception to this was the retention of most of the label with apo A-IV in plasma LDF. When whole plasma was incubated with LDF-associated ${ }^{125}$ I-apo A-IV, only $30 \%$ of radioactivity transferred to HDL, but the specific radioactivity of HDL apo A-IV was $\sim 15$-fold higher than that of LDF apo A-IV, a ratio similar to the one observed when plasma was incubated with "free" radioiodinated apo A-IV.

We also measured the apo A-IV specific radioactivity in TRL, HDL, and LDF after a mixture of lymph and plasma had been incubated with ${ }^{125} \mathrm{I}$-apo A-IV. As shown in Table III the specific radioactivities of apo A-IV present in TRL and in HDL were of a similar order and $\sim 15$-fold higher than that of apo A-IV in LDF.

Further studies were carried out to exclude the possibility that isolated apo A-IV might not exchange as readily with LDF apo A-IV as would native apo A-IV within HDL. We therefore determined the redistribution of ${ }^{125} \mathrm{I}$-labeled apo A-IV after incubation of uniformly labeled HDL with plasma. The specific radioactivities of apo A-IV in HDL and in LDF were determined by excising and counting the gel slice corresponding to apo AIV and by immunoassay of the apo A-IV mass in HDL and LDF (Table III). The ratio of apo A-IV specific radioactivities in HDL/LDF was 15.3 with uniformly labeled HDL. This demonstrates that native apo A-IV was no more freely exchangeable between LDF and HDL than isolated labeled apo A-IV.

In control studies in which ${ }^{125} \mathrm{I}$-apo A-IV associated with either lymph TRL, plasma HDL, or plasma LDF was incubated in buffer $A$, the entire label remained in the chromatographed TRL, HDL fraction, and LDF, respectively.

In vivo metabolism of apo $A-I V$. The results of the five studies are shown in Figs. 3-7, the upper panels showing the redistribution of ${ }^{125} \mathrm{I}$-apo A-IV among TRL, HDL, and LDF and the lower panels the specific radioactivities of apo A-IV in HDL and LDF. Total recovery of radioactivity in plasma $5 \mathrm{~min}$ after the reinjections was $89-98 \%$. Clinical data of the subjects involved in the studies are given in Table IV.

Table III. Specific Radioactivity of Apo A-IV in TRL, HDL, and LDF of Plasma After Incubating Plasma and Lymph with ${ }^{125}$ I-Apo A-IV and After Incubating Plasma with ${ }^{125} I-H D L$

\begin{tabular}{llll}
\hline & \multicolumn{4}{l}{ Specific radioactivity of apo A-IV } \\
\cline { 2 - 4 } Incubation & TRL & HDL & LDF \\
\hline & $c p m / n g$ & $c p m / n g$ & $c p m / n g$ \\
& & & \\
125I-Apo A-IV & & & \\
+ plasma and lymph & $6.24 \pm 2.05^{*}$ & $5.44 \pm 1.18$ & $0.43 \pm 0.09$ \\
${ }^{125}$ I-Apo A-IV + lymph & 5.40 & 6.70 & 0.36 \\
${ }^{125}$ I-HDL + plasma & ND & $45.8 \pm 11.7$ & $3.0 \pm 0.45$ \\
& & & \\
\hline
\end{tabular}

* Means \pm SD of three incubations of a mixture of whole lymph and plasma with ${ }^{125} \mathrm{I}$-apo A-IV and plasma with ${ }^{125} \mathrm{I}-\mathrm{HDL}$.

ND, not determined.
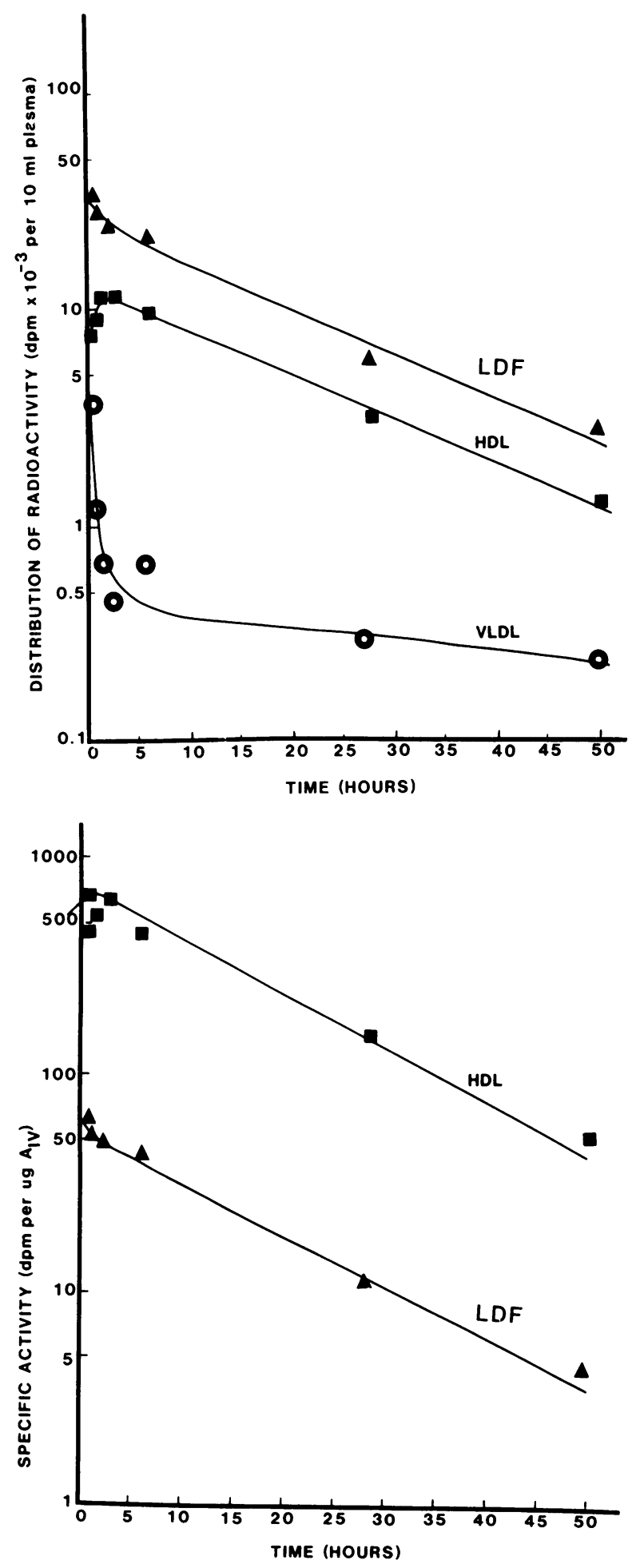

Figure 3. Redistribution of ${ }^{125} \mathrm{I}$-apo A-IV after injection of in vitro labeled ${ }^{125} \mathrm{I}$-apo A-IV lymph TRL into a normolipidemic woman. Top panel shows total radioactivity in VLDL, HDL, and LDF. Bottom panel shows specific radioactivity in apo A-IV in HDL and LDF.

The curves could be resolved into two exponential functions over $48 \mathrm{~h}$. The five studies showed general features in common: (a) ${ }^{125} \mathrm{I}$-apo A-IV transferred to or exchanged with apo A-IV in other apo A-IV containing plasma fractions (TRL or VLDL, HDL, and LDF) irrespective of the nature of the injectate; $(b)$ 

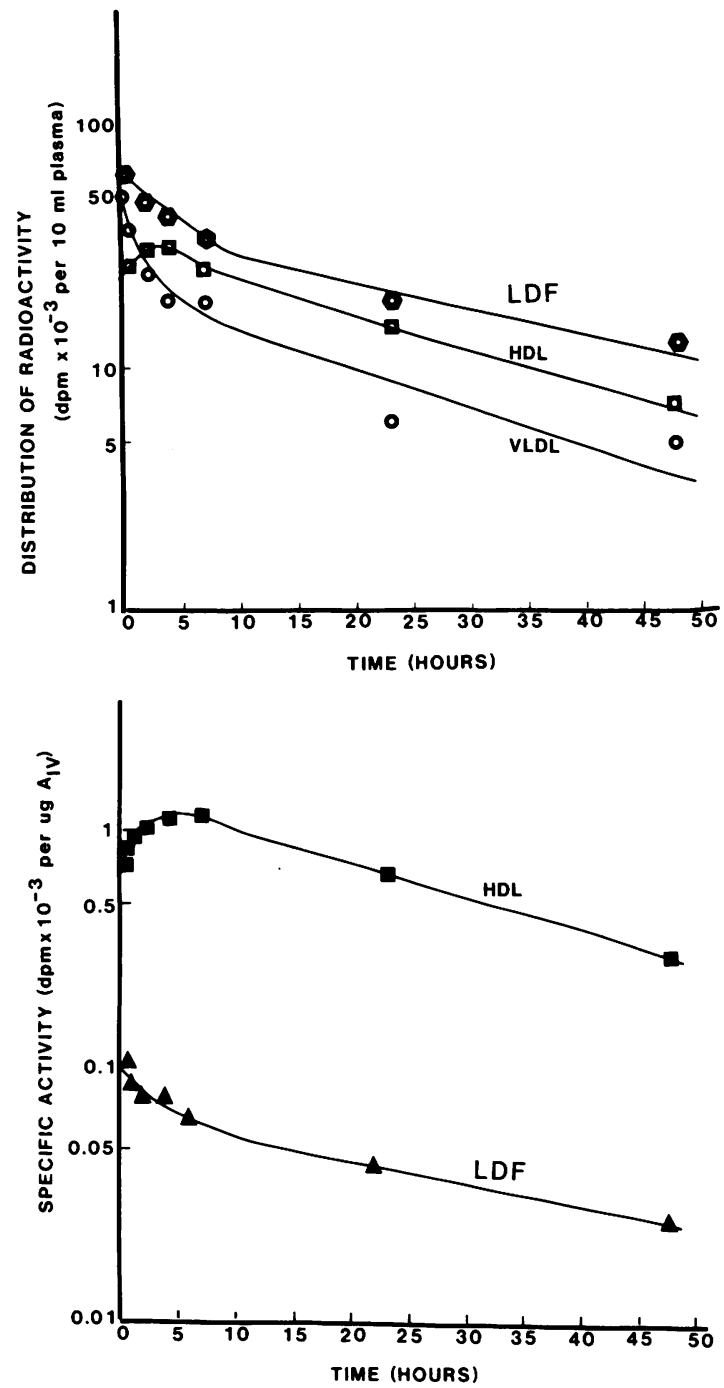

Figure 4. Redistribution of ${ }^{125} \mathrm{I}$-apo A-IV after injection of in vitro labeled ${ }^{125} \mathrm{I}$-apo A-IV plasma TRL into a man with type 5 hyperlipoproteinemia. Top panel shows total radioactivity in VLDL, HDL, and LDF. Bottom panel shows specific radioactivity of apo A-IV in HDL and LDF.

disproportionately more ${ }^{125} \mathrm{I}$-apo A-IV became rapidly associated with HDL than with LDF; $(c)$ the specific radioactivity of apo A-IV was therefore invariably much higher (10-30-fold) in HDL than in LDF; $(d)$ isotopic exchange was attained rapidly with apo A-IV in LDF and appeared slowest for apo A-IV in TRL (VLDL); (e) the specific radioactivity time curves for apo A-IV in HDL and in LDF declined apparently in parallel over the first $48 \mathrm{~h}$, suggesting that the bulk of apo A-IV in LDF did not exchange with that in HDL or at most, very slowly; and $(f)$ the half-life for the decline of the slower, second exponential was of the order of 20-30 h.

\section{Discussion}

As pointed out earlier, a physiological role for apo A-IV remains unknown. The peptide becomes associated with lymph TRL during the secretion of chylomicrons (12) but in human plasma is mainly found unassociated with the major lipoproteins (LDF), with $<5 \%$ of the total present in HDL. This unusual plasma
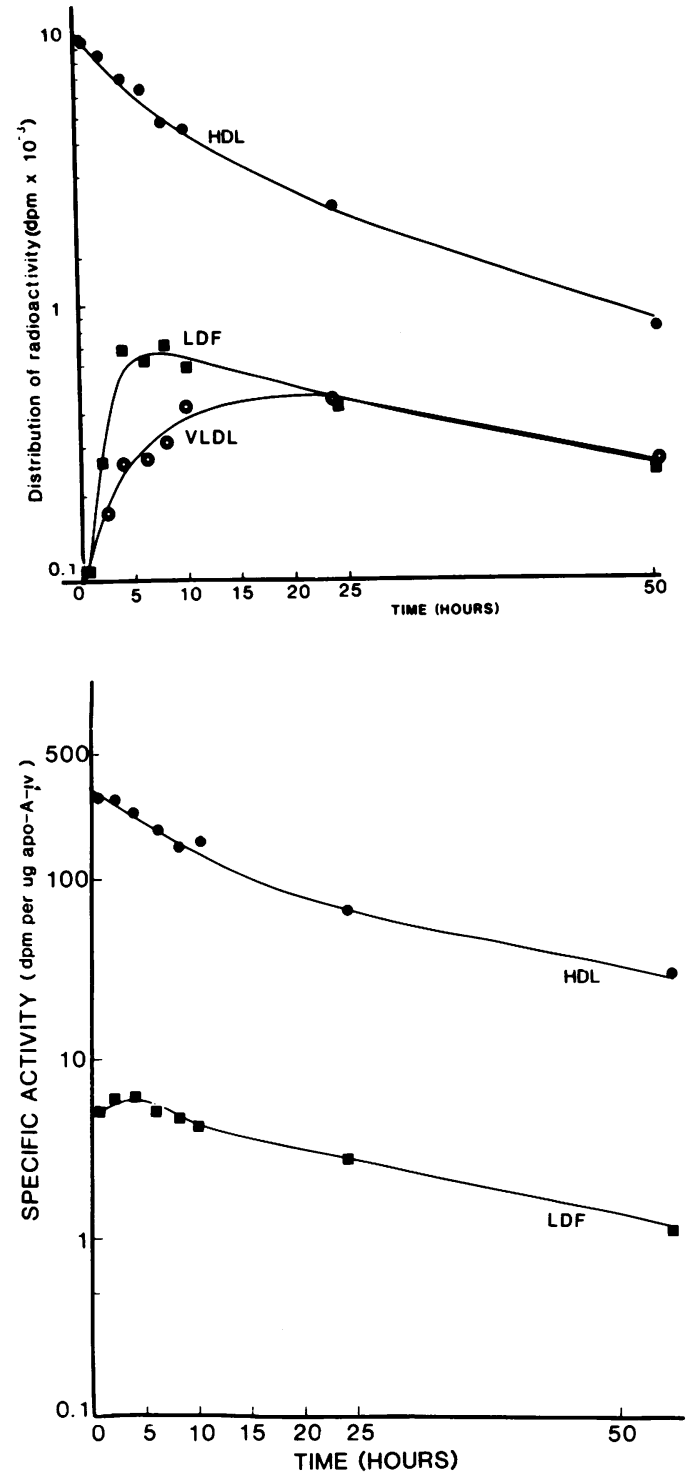

Figure 5. Redistribution of ${ }^{125} \mathrm{I}$-apo A-IV after injection of in vitro labeled ${ }^{125}$ I-apo A-IV plasma HDL into a man with mild hypertriglyceridemia. Top panel shows total radioactivity in VLDL, HDL, and LDF. Bottom panel shows specific radioactivity of apo A-IV in HDL and LDF.

distribution may be the end result of its metabolic fate or representative of its functional role in lipid transport. On the basis of our previous study (11) it seems unlikely that apo A-IV in LDF is structurally distinct from that associated with lipoproteins. However, apo A-IV apparently unassociated with lipoproteins in LDF may exist in a unique association with other proteins, lipids, or other unknown components, differing from its state within HDL or chylomicrons (11). In this study we have further investigated this possibility by examining the ability of labeled apo A-IV to exchange or transfer between the various lipoprotein or nonlipoprotein compartments of plasma and lymph during in vitro incubations and after in vivo infusions.

In vitro studies. After incubating ${ }^{125} \mathrm{I}$-apo A-IV with normal plasma, $72 \%$ of the label was associated with the HDL fraction and only $14 \%$ was not associated with lipoproteins (experiment 1, Table II). This proportioning of label is quite different from 

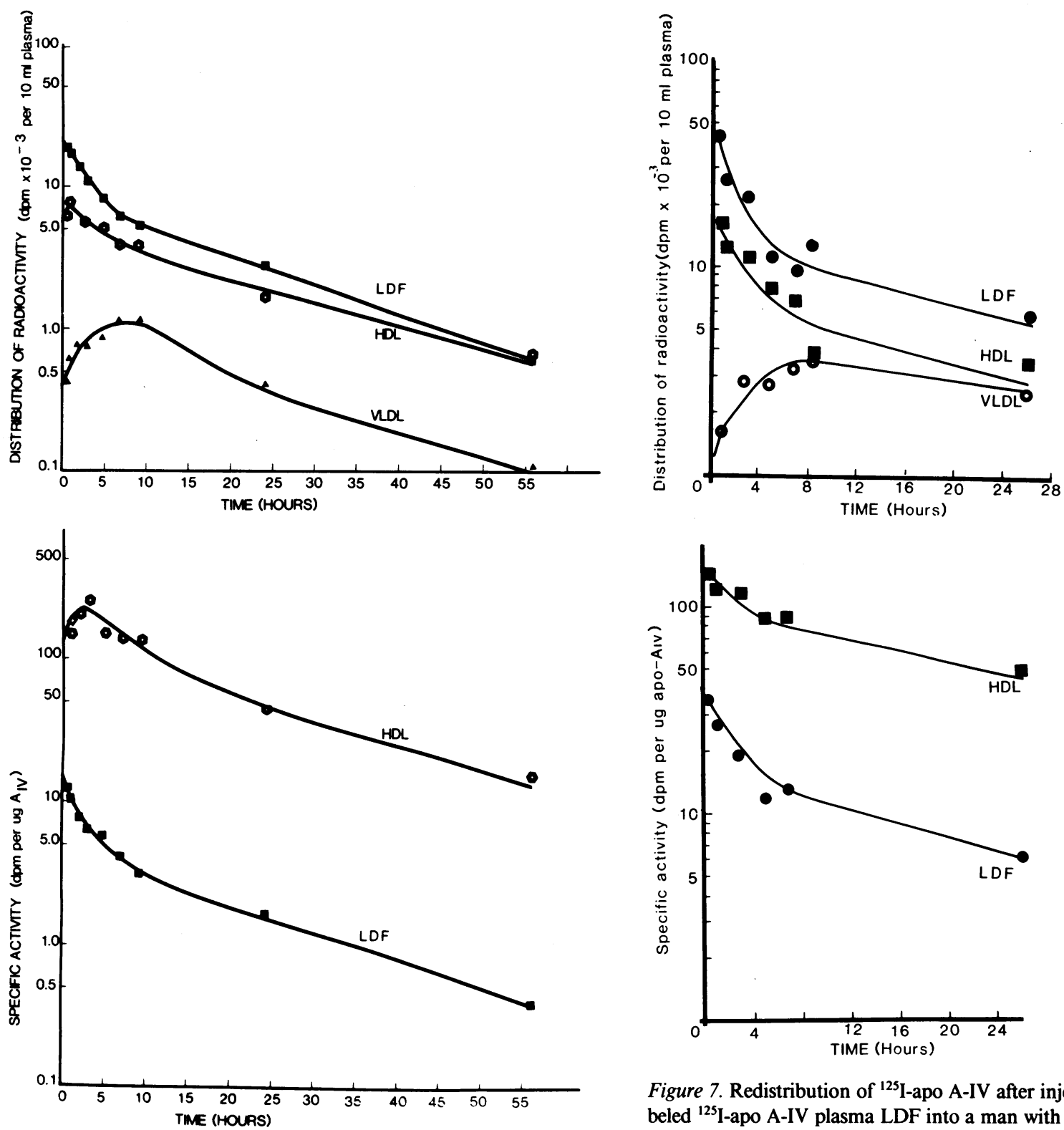

Figure 7. Redistribution of ${ }^{125} \mathrm{I}$-apo A-IV after injection of in vitro labeled ${ }^{125} \mathrm{I}$-apo A-IV plasma LDF into a man with moderate hypertriglyceridemia. Top panel shows total radioactivity in VLDL, HDL, and LDF. Bottom panel shows specific radioactivity of apo A-IV in HDL

Figure 6. Redistribution of ${ }^{125} \mathrm{I}$-apo A-IV after injection of in vitro labeled ${ }^{125} \mathrm{I}$-apo A-IV plasma LDF into a man with combined hyperlipoproteinemia. Top panel shows total radioactivity in VLDL, HDL, and LDF. Bottom panel shows specific radioactivity of apo A-IV in HDL and LDF.

the mass distribution of plasma apo A-IV, in which $>90 \%$ of the apo A-IV is present in LDF. Specific radioactivity determinations after incubation emphasized the fact that the redistribution of ${ }^{125} \mathrm{I}$-apo A-IV was not proportional to the distribution of apo A-IV mass since HDL apo A-IV had a 15-fold higher specific radioactivity than that of LDF apo A-IV. When we incubated ${ }^{125} \mathrm{I}$-apo A-IV with human thoracic lymph (experiment 2) $65 \%$ of apo A-IV was recovered in TRL and $13 \%$ was associated in LDF; the specific radioactivity of TRL apo A-IV was also 15-fold higher than that of LDF apo A-IV. By contrast, the specific radioactivities of TRL apo A-IV and HDL apo A-IV became similar, indicating that the distribution of ${ }^{125} \mathrm{I}$-apo AIV between TRL and HDL was proportional to the distribution of apo A-IV mass between these two fractions, but not between

Table IV. Clinical Data of Subjects Participating in the In Vivo Studies of Apo A-IV Metabolism

\begin{tabular}{|c|c|c|c|c|}
\hline \multirow[b]{2}{*}{ Subject } & \multicolumn{2}{|l|}{ Plasma } & \multirow[b]{2}{*}{ Plasma apo A-IV } & \multirow[b]{2}{*}{ Injected sample } \\
\hline & Triglyceride & Cholesterol & & \\
\hline & $m g / d l$ & $m g / d l$ & $m g / d l$ & \\
\hline $1(\mathrm{~F})$ & 130 & 170 & 14.0 & Lymph TRL \\
\hline $2(\mathrm{M})$ & 1,250 & 261 & 17.6 & Plasma TRL \\
\hline $3(\mathrm{M})$ & 220 & 250 & 20.2 & Plasma HDL \\
\hline $4(\mathrm{M})$ & 414 & 306 & 18.5 & Plasma LDF \\
\hline $5(M)$ & 525 & 295 & 22.4 & Plasma LDF \\
\hline
\end{tabular}

F, female; $M$, male. 
apo A-IV in TRL and LDF or between apo A-IV in HDL and LDF.

When labeled apo A-IV in LDF was incubated with plasma, most labeled apo A-IV remained in LDF (experiment 6), although the specific radioactivity of HDL apo A-IV was also 15fold higher than that of LDF apo A-IV. This interesting result together with experiment 1 indicates that most of the unlabeled mass of apo A-IV in LDF is refractive to free exchange with labeled apo A-IV (experiment 1) or to free exchange or transfer with apo A-IV in HDL or TRL. Nevertheless a small fraction of the apo A-IV in LDF was exchangeable with these lipoproteins because the labeled apo A-IV which became associated with the LDF was capable of reassociating with $\mathrm{HDL}$, and vice versa. These findings suggest the possibility that, in LDF, apo A-IV exists in two pools, of which one is exchangeable while the other is independent of the redistribution system involving plasma or lymph lipoproteins.

The possibility that the isolated or radioiodinated apo A-IV preparations contained significant proportions of nonexchangeable material due to denaturation is unlikely for several reasons. First, the source of the apolipoprotein was lymph chylomicrons, and the peptide was immunoaffinity purified in contrast to other isolation procedures involving lengthy exposures to denaturing reagents. Second, this apo A-IV showed a similar isoprotein pattern by two-dimensional gel electrophoresis (11) to that obtained with delipidated whole lymph chylomicrons. Third, the labeled apo A-IV completely equilibrated between TRL and HDL. Fourth, the physical-chemical properties described above show no apparent evidence of denaturation after isolation or radioiodination of apo A-IV.

In a previous study, we showed that in fact apo A-IV does exist in LDF in a heterogeneous state in association with lipids and other apolipoproteins (11). Attempts to isolate free apo AIV from LDF and lymph TRL by immunoabsorbance affinity chromatography were successful only with delipidated lymph TRL apolipoprotein. With LDF, even after delipidation using chloroform/methanol and ether, apo A-IV could not be separated from a complex involving apo A-IV and A-I and a protein of $M_{\mathrm{r}} 59,000$. Lipid, especially cholesteryl ester, was also associated with this complex, suggesting that even within LDF apo A-IV exists in a lipoproteinlike species (11). SDS disk gel electrophoresis of this complex from LDF showed a similar protein profile to that obtained from nondelipidated LDF after immunoabsorbance chromatography (11). These results strongly suggest that apo A-IV exists in LDF in some tight complex involving protein-protein or protein-lipid bonds that do not permit free exchange of apo A-IV.

In vivo studies. These studies reinforce the conclusions drawn from the incubations. However, in comparing the two sets of data it must be remembered that the design of the in vivo studies was closer to that of in vitro experiments 4-6 (Table II) than to that of experiments $1-3$, since the injectates contained ${ }^{125} \mathrm{I}$-apo A-IV that had been preequilibrated in vitro with the test lipoprotein or LDF fraction. As already discussed, such preincubation allows for lesser subsequent exchange than when free ${ }^{125} \mathrm{I}$-apo A-IV is tested.

Nevertheless, each of the in vivo studies was consistent with the in vitro data in showing rapid exchange of ${ }^{125} \mathrm{I}$-apo A-IV among plasma apo A-IV containing fractions and the failure of isotopic equilibrium to occur between apo A-IV in HDL and in LDF. Indeed the higher "final" specific radioactivity for apo A-IV in HDL was similar in both the in vitro and in vivo ex- periments. It is therefore clear that only a minor fraction of apo A-IV in LDF is exchangeable with other apo A-IV in plasma. As indicated above, this is consistent with our previous observation (11) that apo A-IV exists in "minilipoprotein" form in more than one complex, with that in HDL differing from that in LDF.

In both the in vitro and in vivo experiments, the amount of ${ }^{125} \mathrm{I}$-apo A-IV that became associated with HDL was disproportionately high in relation to its mass distribution. In the in vivo studies, most labeled apo A-IV remained with the HDL when HDL was reinjected (Fig. 5); less associated with HDL when LDF or TRL were reinjected. HDL therefore appears to have a particularly high affinity for at least one apo A-IV complex which, based on the evidence, has a far greater rate of metabolism than the less freely exchangeable apo A-IV complex that represents a major form in LDF. The apparently similar rates of removal of ${ }^{125} \mathrm{I}$-apo A-IV, after the initial equilibration period (of the exchangeable pool) within the TRL (VLDL), HDL, and LDF, probably reflects the metabolism of one common complex of apo A-IV.

These studies do not indicate whether both apo A-IV complexes in LDF participate in the rapid exchange with TRL and LDF or whether apo A-IV in one complex is more refractory to exchange than is the other complex. Possibly two complexes have different physiological functions. The exchangeable fraction may derive from triglyceride transport from the gut and is readily dissociated from lymph chylomicrons within plasma. This is consistent with several observations that apo A-IV is synthesized at least in part in the small intestine and becomes incorporated into lymph chylomicrons during the absorption of fat (12). It is not clear why HDL should have such a high affinity for apo AIV, other than this is not unique for this chylomicron peptide. However, in a previous study in the rat (3), we noted an even greater transfer of chylomicron ${ }^{125}$ I-apo A-IV to HDL, consistent with the relatively greater mass of apo A-IV in rat HDL. Furthermore, a proportion of LDF ${ }^{125} \mathrm{I}$-apo A-IV, derived initially from chylomicrons, transferred later to HDL, suggesting that in the rat there may be greater homogeneity of apo A-IV complexes between HDL and LDF than we have now observed in humans.

In addition to the clear involvement of apo A-IV in dietary triglyceride transport, there is increasing evidence that the metabolism of apo A-IV may be related to cholesterol metabolism. Delamatre and Roheim (8) recently found that dietary cholesterol led to redistribution of apo A-IV from HDL into the lipoproteinfree fraction in rats. Furthermore both lecithin cholesterol acyltransferase (9) and lipoprotein lipase (22) activity also appear to influence the distribution of apo A-IV, at least in the rat. It is also of interest that LCAT activity from human plasma can be moderately stimulated by apo A-IV (23) though only $25 \%$ as effectively as a corresponding amount of apo A-I.

Finally, the large pool of apo A-IV present in the fraction of plasma of $d>1.21 \mathrm{~g} / \mathrm{ml}$, which has now been shown to exist in discrete lipid-protein complexes (11), may have several metabolic functions. In recent studies in this laboratory (Fidge, N. H., and P. J. Nestel, unpublished observations), it was found that radiolabeled apo A-IV was able to transfer from the plasma into the lymph compartment of rats where it became associated with chylomicrons after fat feeding. This shows that apolipoproteins can move freely between lymph and plasma and transfer to chylomicrons after their assembly and secretion from enterocytes. One other possible explanation for the unusual distribution of apo A-IV is that the peptide may have other metabolic func- 
tions independent of fat transport. These possibilities await more detailed information about the tissue distribution of apo A-IV and those factors that regulate its cellular synthesis and removal within the body.

\section{Acknowledgments}

The authors are grateful to Margaret O'Connor and Hubert Edelsbacher for expert technical assistance.

We thank the National Health \& Medical Research Council (Australia) for grants supporting this work.

\section{References}

1. Weisgraber, K. H., T. P. Bersot, and R. W. Mahley. 1978. Isolation and characterization of an apoprotein from the $d<1.006$ lipoproteins of human and canine lymph homologous with the rat A-IV apoprotein. Biochem. Biophys. Res. Commun. 85:287-292.

2. Swaney, J. B., F. Braithwaite, and H. A. Eder. 1977. Characterization of the apolipoproteins of rat plasma lipoproteins. Biochemistry. 16:271-278.

3. Fidge, N. H. 1980. The redistribution and metabolism of iodinated apolipoprotein A-IV in Rats. Biochim. Biophys. Acta 619:129-141.

4. Beisiegel, U., and G. Utermann. 1979. An apolipoprotein homologue of rat apolipoprotein A-IV in human plasma. Isolation and partial characterization. Eur. J. Biochem. 93:601-608.

5. Utermann, G., and U. Beisiegel. 1979. Apolipoprotein A-IV: a protein occurring in human mesenteric lymph chylomicrons and free in plasma. Isolation and quantification. Eur. J. Biochem. 99:333-343.

6. Bar-On, H., P. S. Roheim, and H. A. Eder. 1976. Serum lipoproteins and apolipoproteins in rats with streptozotocin induced diabetes. J. Clin. Invest. 57:714-721.

7. Van Lenten, B. J., and P. S. Roheim. 1982. Changes in the concentrations and distributions of apolipoproteins of the aging rat. J. Lipid Res. 23:1187-1195.

8. Delamatre, J. G., and P. S. Roheim. 1983. The response of apolipoprotein A-IV to cholesterol feeding in rats. Biochim. Biophys. Acta. 751:210-217.

9. Delamatre, J. G., C. A. Hoffmeier, A. G. Lacko, and P. S. Roheim. 1983. Distribution of apolipoprotein A-IV between the lipoprotein and the lipoprotein-free fractions of rat plasma: possible role of lecithin: cholesterol acyltransferase. J. Lipid Res. 24:1578-1585.
10. Bisgaier, C., O. Sachdev, L. Megna, R. Glickman. 1983. Plasma distribution of human apo A-IV. Arteriosclerosis. 3:497a.

11. Ohta, T., N. H. Fidge, and P. J. Nestel. 1984. Characterization of apolipoprotein AIV complexes and AIV isoforms in human lymph and plasma lipoproteins. J. Biol. Chem. 259:14888-14893.

12. Green, P. H. R., R. M. Glickman, J. W. Riley, and E. Quinet. 1980. Human apolipoprotein A-IV. Intestinal origin and distribution in plasma. J. Clin. Invest. 65:911-919.

13. Nestel, P. J., N. H. Fidge, and M. H. Tan. 1982. Increased lipoprotein-remnant formation in chronic renal failure. N. Engl. J. Med. 307:329-333.

14. Wu, A. L., and H. G. Windmueller. 1979. Relative contributions by liver and intestine to individual plasma apolipoprotein in the rat. $J$. Biol. Chem. 254:7316-7322.

15. Fidge, N. H., and P. Poulis. 1975. Studies on the metabolism of rat serum very low density lipoproteins. J. Lipid Res. 16:367-378.

16. Cuatrecasas, P. 1970. Protein purification by affinity chromatography. Derivatizations of agarose and polyacrylamide beads. J. Biol. Chem. 245:3059-3065.

17. McFarlane, A. S. 1968. Efficient trace labeling of proteins with iodine. Nature. (Lond.). 182:53.

18. Weber, K., and M. Osborn. 1975. Protein and sodium dodecylsulfate. Molecular weight determination on polyacrylamide gels and related procedures. In The Protein. H. Neurath and R. L. Hill, editors. Academic Press, New York. 179-223.

19. Gidez, L. I., J. B. Swaney, and S. Murnane. 1977. Analysis of rat serum apolipoproteins by isoelectric focusing. I. Studies on the middle molecular weight subunits. J. Lipid Res. 18:59-68.

20. Huff, M. W., N. H. Fidge, P. J. Nestel, T. Billington, and B. Watson. 1981. Metabolism of C-apolipoproteins: kinetics of C-II, C-III, and $\mathrm{C}-\mathrm{III}_{2}$, and VLDL-apolipoprotein $\mathrm{B}$ in normal and hyperlipoproteinemic subjects. J. Lipid Res. 22:1235-1246.

21. Havel, R. J., H. A. Eder, and J. H. Bragdon. 1955. The distribution and chemical composition of ultracentrifugally separated lipoproteins in human serum. J. Clin. Invest. 34:1345-1353.

22. Chuang, M.-Y., M. Lefevre, and P. S. Roheim. 1984. Influence of lipolytic activity on the redistribution of rat plasma apo A-IV. Arteriosclerosis. 4:519a. (Abstr.)

23. Chen, C.-H., and J. J. Albers. 1984. Activation of lecithin-cholesterol acyltransferase by apoprotein E and A-VI. Arteriosclerosis. 4: 519a. (Abstr.) 